\title{
Outage Analysis of Distributed CDD Systems with Mixture Interference
}

\author{
Kyeong Jin Kim, Hongwu Liu, Marco Di Renzo, Theodoros A. Tsiftsis, \\ Philip V. Orlik, and H. Vincent Poor
}

\begin{abstract}
In this paper, a cooperative single carrier system comprising multiple cooperating remote radio heads and a single interferer operating in the presence of co-existing line-of-sight and non-line-of-sight paths is investigated. Distributed cyclic delay diversity is employed as the transmit diversity scheme for cyclic-prefixed single carrier transmissions over independent but non-identically distributed frequency selective fading channels. The main focus of this paper is to investigate the achievable diversity gain in the interference-limited and noise-limited regions. In contrast to the outage probability in the noise-limited region, it is shown that the diversity gain is not achievable in the interference-limited region. To justify this finding, the outage probability is derived first, and then verified by link-level simulations.
\end{abstract}

\section{Index Terms}

Distributed cyclic delay diversity, line-of-sight and non-line-of-sight paths, cyclic-prefixed single carrier transmission, interferers, outage probability, diversity gain.

\section{INTRODUCTION}

When channel state information (CSI) is available at the transmitter side (CSIT), an increased receive signalto-noise ratio (SNR) can be obtained at the receiver side by the use of a transmit diversity scheme. However, acquiring exact CSI is a challenging task in distributed systems. As the practical transmit diversity scheme that does not require CSIT via explicit channel feedback from the receiver side, distributed cyclic delay diversity (dCDD) has been proposed for cyclic-prefixed single carrier (CP-SC) transmissions [1], [2]. The dCDD scheme is based on the recognition that it improves the reliability of a message by transmitting over multiple communication channels having different channel characteristics. The conventional CDD, which is deployed in a single transmitter, has been employed in orthogonal frequency division multiplexing (OFDM)-based wireless systems such as [3], [4], and [5]. Several works [6]-[9] have also considered the use of the conventional CDD scheme for CP-SC transmissions. Only a recent work [1] proposes a dCDD scheme for a cooperative system with a set of distributed single-antenna

K. J. Kim and P. V. Orlik are with Mitsubishi Electric Research Laboratories (MERL), Cambridge, MA, USA.

H. Liu is with Shandong Jiaotong University, Jinan, China.

M. D. Renzo is with the Laboratoire des Signaux et Systèmes, CNRS, CentraleSupélec, Univ Paris Sud, Université Paris-Saclay, 3 rue Joliot Curie, Plateau du Moulon, 91192, Gif-sur-Yvette, France.

T. A. Tsiftsis is with the School of Electrical \& Information Engineering, Jinan University, Zhuhai, China.

H. V. Poor is with the Department of Electrical Engineering, Princeton University, Princeton, NJ, USA.

This work was supported in part by the U.S. National Science Foundation under Grants CCF-093970 and CCF-1513915. 
equipped transmitters. Using the conventional CDD, an intersymbol interference (ISI)-free single-input and singleoutput (SISO) channel can be obtained from the multiple-input and single-output channel. For CP-SC transmissions, the equivalent SISO channel matrix can be expressed as a right circulant matrix, which provides a sufficient condition in determining the maximum number of single-antenna equipped transmitters for dCDD. That is, the maximum number of transmitters for $\mathrm{dCDD}$ is mainly determined by the size of the transmission symbol block and the maximum number of multipath components between two nodes in the system. Since the dCDD protocol allows as many as there are transmitters being used to the extent supported by dCDD, a full diversity gain can be achieved as those of [10], [11] with an accompanying higher coding gain. This finding overcomes the result of [7] that the full diversity gain is not achievable at a full transmission rate.

Several recent works [12]-[15] consider the co-existence of line-of-sight (LoS) and non-line-of-sight (nLoS) paths between base stations (BSs) and a user terminal (UT) especially for mobile communications. Similar to this setting, as a system model, we assume a communication cell, wherein one interferer is assumed to be present in the environment coexisting with $\mathrm{nLoS}$ and LoS paths. Based on this new channel model, this paper analyzes the outage probability of the $\mathrm{dCDD}$ based CP-SC system for independent but non-identically distributed (i.n.i.d.) frequency selective fading channels.

In contrast to existing work, we can summarize our main contributions as follows.

- Since we consider a limited number of remote radio heads (RRHs) for dCDD in the presence of a single interferer, we use a mathematical analysis fit to finite-sized cooperative systems. For this finite-sized cooperative system, we investigate the impact of interference on the outage probability.

- In contrast to the work [1], we employ a different channel model, which is somewhat similar to [12]-[15]. The co-existence of LoS and nLoS paths is modeled by using a fading time-share factor [16] that follows a binary Bernoulli process. This can be recognized as an ergodic factor obtained from long observations.

- We provide an analytical framework taking into account a different degree of RRH cooperation via dCDD, frequency selectivity, and interference over the co-existing LoS and nLoS paths. For this new system setting, a new expression for the cumulative distribution function (CDF) is derived for the computation of the outage probability.

- We analyze the achievable diversity gain in the interference-limited and noise-limited regions. From this focus, the limit on the outage probability in the interference-limited region is analyzed and justified.

\section{A. Notation}

$\mathbb{C}$ denotes the set of complex numbers; $\left\|\boldsymbol{a}_{n}\right\|$ denotes the Euclidean norm of a vector $\boldsymbol{a}_{n} ; \mathcal{C N}\left(\mu, \sigma^{2}\right)$ denotes the circularly symmetric complex Gaussian distribution with mean $\mu$ and variance $\sigma^{2} ; B(a)$ denotes the binary Bernoulli process with success probability $a ; F_{\varphi}(\cdot)$ and $f_{\varphi}(\cdot)$, respectively, denote the CDF and probability density function (PDF) of the random variable (RV) $\varphi$; and $E\{\cdot\}$ denotes expectation. In addition, $\left(\begin{array}{l}n_{1} \\ n_{2}\end{array}\right) \triangleq \frac{n_{1} !}{n_{2} !\left(n_{1}-n_{2}\right) !}$ denotes the binomial coefficient. The length of a vector $\boldsymbol{a}$ is denoted by $\mathbb{L}(\boldsymbol{a})$. 


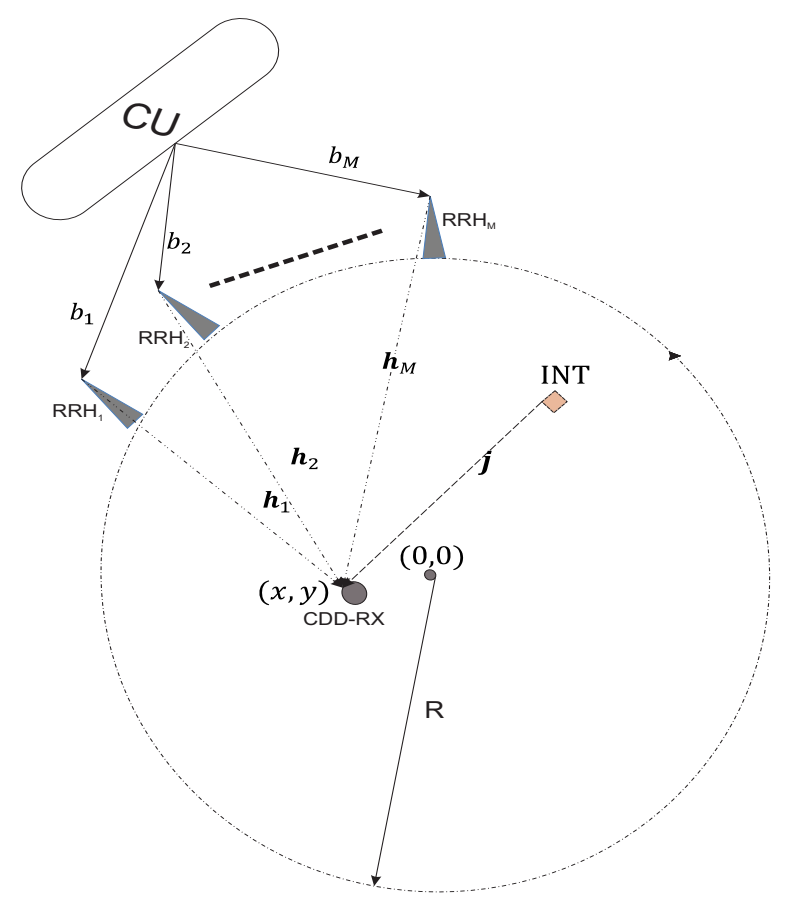

Fig. 1. Illustration of the considered dCDD-based cooperative CP-SC system in the presence of a single interferer (INT).

\section{System And Channel Model}

Fig. 1 illustrates the considered cooperative CP-SC system, which is composed of a control unit (CU) connected to $M$ single-antenna equipped RRHs, $\left\{\mathrm{RRH}_{m}\right\}_{m=1}^{M}$, via dedicated highly reliable backhaul connections, $\left\{b_{m}\right\}_{m=1}^{M}$. The CU forms an information signal and controls RRHs to transmit the information signal simultaneously using dCDD [1], so that each of the RRHs requires only simple hardware and transmission power in communicating with the receiver (CDD-RX). A circularly shaped cell with a radius $R$ is formed with respect to the RRHs, within which a single-antenna equipped interferer (INT) exists. Perfect synchronization among RRHs is available by the time stamp provided by a Global Navigation Satellite System (GNSS) (e.g., GPS, Galileo, Glonass, etc.), so that RRHs can transmit the same information signal at the same time when they are chosen for dCDD. Due to use of half-duplex transceivers in the nodes, all the nodes are allowed to transmit or receive at any given time.

Compared with large-scale systems [12]-[14], this paper investigates only a finite-sized cooperative system comprising a fixed number of RRHs for dCDD in the presence of a single interferer. Since dCDD is optimized through channels connecting the CDD-RX, the set of frequency selective fading channels from the RRHs to the CDD-RX is assumed to be composed of LoS paths, whereas a channel from the interferer to the CDD-RX is assumed to be comprised of LoS and nLoS paths [12]-[15] due to its possible random location.

By employing an appropriate channel sounding scheme or channel reciprocity [17], [18], we further assume that the CDD-RX is able to know the maximum number of multipath components over the channels from RRHs to the CDD-RX. 


\section{A. Channels from RRHs to the CDD-RX}

The multipath channel, $\boldsymbol{h}_{k}$, from the $k$ th RRH to the CDD-RX is given by

$$
\boldsymbol{h}_{k}=\sqrt{d_{k}^{-\epsilon_{\mathrm{L}}}} \tilde{\boldsymbol{h}}_{k}
$$

where $\tilde{\boldsymbol{h}}_{k}$ denotes frequency selective fading for $\boldsymbol{h}_{k}$ with $N_{h} \triangleq \mathbb{L}\left(\tilde{\boldsymbol{h}}_{k}\right)$ LoS multipath components; $d_{k}$ denotes the distance from the $k$ th RRH to the CDD-RX; and $\epsilon_{\mathrm{L}}$ denotes the path loss exponent of the LoS path. Due to a different distance from a particular RRH to the CDD-RX, we assume i.n.i.d. frequency selective fading over the channels from RRHs to the CDD-RX.

\section{B. Channel from the interferer to the CDD-RX}

The channel from the interferer to the CDD-RX is given by

$$
\boldsymbol{j}=\mathbb{I}_{j, \mathrm{~L}} \sqrt{\left(d_{J}\right)^{-\epsilon_{\mathrm{L}}}} \tilde{\boldsymbol{j}}_{\mathrm{L}}+\mathbb{I}_{j, \mathrm{~nL}} \sqrt{\left(d_{J}\right)^{-\epsilon_{\mathrm{nL}}}} \tilde{\boldsymbol{j}}_{\mathrm{nL}}
$$

where $\tilde{\boldsymbol{j}}_{\mathrm{L}}$ and $\tilde{\boldsymbol{j}}_{\mathrm{nL}}$, respectively, identify frequency selective fading for $\operatorname{LoS}$ and $\mathrm{nLoS}$ paths with $N_{j, \mathrm{~L}} \triangleq \mathbb{L}\left(\tilde{\boldsymbol{j}}_{\mathrm{L}}\right)$ and $N_{j, \mathrm{~nL}} \triangleq \mathbb{L}\left(\tilde{j}_{\mathrm{nL}}\right)$. The distance from the interferer to the CDD-RX is assumed to be $d_{J}$. In addition, $\epsilon_{\mathrm{nL}}$ denotes the path loss exponent of the nLoS path. Complementary indicator functions $\mathbb{I}_{j, \mathrm{~L}}$ and $\mathbb{I}_{j, \mathrm{~nL}}$ are used to model co-existing LoS and nLoS paths by a time-share factor, $0 \leq \mathcal{F} \leq 1$, as follows:

$$
\mathbb{I}_{j, \mathrm{~nL}} \sim B(\mathcal{F}) \text { and } \mathbb{I}_{j, \mathrm{~L}} \triangleq 1-\mathbb{I}_{j, \mathrm{~nL}} \sim B(1-\mathcal{F})
$$

which specifies that for a fraction of time $\mathcal{F}$, a channel $j$ introduces the nLoS path with frequency selective fading, whereas for a fraction of time $1-\mathcal{F}$, the channel $j$ introduces the LoS path with independent frequency selective fading. A constant time-share for $\operatorname{LoS}$ and $\mathrm{nLoS}$ paths is assumed during transmissions over the channels from the interferer to the CDD-RX.

\section{C. dCDD for CP-SC Transmissions}

We assume that single carrier transmissions with the use of the cyclic prefix [10] are used by every node in the system. To remove ISI due to a multipath channel, the last $N_{p}$ modulation symbols from a transmission symbol block $s \in \mathbb{C}^{B \times 1}$ are appended to the front of $s$ [1]. The size of the transmission symbol block $s$ is denoted by $B$. We assume that $E\{s\}=\mathbf{0}$ and $E\left\{\|s\|^{2}\right\}=\boldsymbol{I}_{B}$. To make an ISI-free right circulant channel matrix, the CP length, $N_{p}$, and the $i$ th CDD delay, $\Delta_{i}$, need to be designed efficiently by the following two conditions [1]:

$$
N_{p} \geq \max \left(N_{h, m}\right) \text { and } \Delta_{i}=(i-1) N_{p} .
$$

Based on (4), the maximum allowable number of RRHs for dCDD is determined by

$$
K=\left\lfloor B / N_{p}\right\rfloor
$$

where $\lfloor\cdot\rfloor$ denotes the floor function. In this paper, we assume that there are only $M$ RRHs, with $M \leq K$, so that every RRH is available for dCDD operation. With this condition, the received signal at the CDD-RX, after removing the $\mathrm{CP}$ signal, is given by

$$
\boldsymbol{r}=\sum_{m=1}^{M} \sqrt{P_{s} d_{m}^{-\epsilon_{\mathrm{L}}}} \tilde{\boldsymbol{H}}_{m} \tilde{\boldsymbol{s}}_{m}+\sqrt{P_{J}} \boldsymbol{J} \boldsymbol{x}_{J}+\boldsymbol{z}_{R}
$$


where $\tilde{\boldsymbol{s}}_{m} \triangleq \boldsymbol{P}_{B}^{\Delta_{k}} \boldsymbol{s}_{m}$, the transmission power allocated at all the RRHs is $P_{s}, \tilde{\boldsymbol{H}}_{m}$ is the right circulant matrix specified by the $m$ th LoS channel $\tilde{\boldsymbol{h}}_{m}$, and $\boldsymbol{P}_{B}^{\Delta_{m}}$ is the permutation shifting matrix ${ }^{1}$, which is obtained from the identity matrix $\boldsymbol{I}_{B}$ by circularly shifting down by $\Delta_{m}$. The additive noise over the desired channels is denoted by $\boldsymbol{z}_{R} \sim \mathcal{C N}\left(\mathbf{0}, \sigma_{z}^{2} \boldsymbol{I}_{B}\right)$. The CDD-RX does not experience interference from simultaneous transmission from the RRHs selected for dCDD operation, whereas the interferer interferes with the CDD-RX since it is not controlled by the CU. The matrix $\boldsymbol{J}$ is also right circulant which is mainly specified by $\boldsymbol{j}$. The interfering symbol block from the interferer is given by $\boldsymbol{x}_{J}$ with $E\left\{\boldsymbol{x}_{J}\right\}=\mathbf{0}$ and $E\left\{\boldsymbol{x}_{J} \boldsymbol{x}_{J}^{H}\right\}=\boldsymbol{I}_{B}$. The transmission power allocated to the interferer is denoted by $P_{J}$.

\section{Performance Analysis}

From (6), the signal-to-interference-pus-noise ratio (SINR) measured at the CDD-RX is given by

$$
\gamma_{\mathrm{SINR}} \triangleq \frac{\sum_{m=1}^{M} \tilde{\alpha}_{h, m}\left\|\tilde{\boldsymbol{h}}_{m}\right\|^{2}}{P_{J}\|\boldsymbol{j}\|^{2}+\sigma_{z}^{2}}=\frac{S}{J+\sigma_{z}^{2}}
$$

where $\tilde{\alpha}_{h, m} \triangleq P_{s}\left(d_{m}\right)^{-\epsilon_{\mathrm{L}}}, S \triangleq \sum_{m=1}^{M} \tilde{\alpha}_{h, m}\left\|\tilde{\boldsymbol{h}}_{m}\right\|^{2}$, and $J \triangleq P_{J}\|\boldsymbol{j}\|^{2}$. Note that by using the QRD-M detector [19], we can obtain (7). To understand the statistical properties of the SINR, we need to prepare the distributions of a channel power in the presence of coexisting LoS and nLoS paths.

\section{A. Distributions relevant to co-existing LoS and nLoS paths}

Proposition 1: Due to co-existing independent $\operatorname{LoS}$ and $\mathrm{nLoS}$ paths, the CDF of the RV $J$, the received interference power at the CDD-RX, is given by

$$
F_{J}(x)=1-(1-\mathcal{F}) \Gamma_{u}\left(N_{j, \mathrm{~L}}, \frac{x}{\tilde{\alpha}_{j, L}}\right) / \Gamma\left(N_{j, \mathrm{~L}}\right)+\mathcal{F} \Gamma_{u}\left(N_{j, \mathrm{~nL}}, \frac{x}{\tilde{\alpha}_{j, n L}}\right) / \Gamma\left(N_{j, \mathrm{~nL}}\right)
$$

where $\tilde{\alpha}_{j, L} \triangleq P_{J}\left(d_{J}\right)^{-\epsilon_{\mathrm{L}}}, \tilde{\alpha}_{j, n L} \triangleq P_{J}\left(d_{J}\right)^{-\epsilon_{\mathrm{nL}}}$, and $\Gamma(\cdot)$ and $\Gamma_{u}(\cdot, \cdot)$ denote the complete gamma and upper incomplete gamma functions, respectively.

Proof: Using the properties of binary Bernoulli process and frequency selective fading channels, we can easily verify (8).

Similarly, the PDF of $J$ is given by

$$
f_{J}(x)=(1-\mathcal{F}) \frac{e^{-x / \tilde{\alpha}_{j, L}} x^{N_{j, \mathrm{~L}}-1}}{\Gamma\left(N_{j, \mathrm{~L}}\right)\left(\tilde{\alpha}_{j, L}\right)^{N_{j, \mathrm{~L}}}}+\mathcal{F} \frac{e^{-x / \tilde{\alpha}_{j, n L}} x^{N_{j, \mathrm{~nL}}-1}}{\Gamma\left(N_{j, \mathrm{~nL}}\right)\left(\tilde{\alpha}_{j, n L}\right)^{N_{j, \mathrm{~nL}}}} .
$$

According to [20], the CDF of the RV $S$ is given by

$$
F_{S}(x)=\sum_{m=1}^{M} \sum_{j=1}^{N_{h, m}}(-1)^{m} \theta_{m, j}\left(\tilde{\alpha}_{h, m}\right)^{j}\left[1-\sum_{l=0}^{j-1} \frac{\left(\tilde{\alpha}_{h, m}\right)^{-l}}{\Gamma(l+1)} x^{l} e^{-\frac{x}{\alpha_{h, m}}}\right]
$$

with $\theta_{k, j}$ defined by

$$
\theta_{m, j} \triangleq \frac{(-1)^{N_{h, m}}}{\left(\tilde{\alpha}_{h, m}\right)^{N_{h, m}}} \sum_{X(m, j)} \prod_{k=1, k \neq m}^{M}\left(\begin{array}{c}
N_{h, k}+q_{k}-1 \\
q_{k}
\end{array}\right) \frac{\left(\tilde{\alpha}_{h, k}\right)^{q_{k}}}{\left(1-\frac{\tilde{\alpha}_{h, k}}{\tilde{\alpha}_{h, m}}\right)^{N_{h, k}+q_{k}}}
$$

\footnotetext{
${ }^{1}$ To achieve an ISI-free right circulant matrix, $\boldsymbol{P}_{B}^{\Delta_{m}}$ also needs to be right circulant, which makes the product of the two matrices $\tilde{\boldsymbol{H}}_{m} \boldsymbol{P}_{B}^{\Delta}$ right circulant.
} 
where $X(m, j)$ denotes a set of $M$-tuples satisfying the following condition:

$$
X(m, j) \triangleq\left\{\left(q_{1}, \ldots, q_{M}\right): \sum_{k=1}^{M} q_{k}=N_{h, m}-j \text { with } q_{i}=0\right\} .
$$

\section{B. Distributions of the SINR}

The CDF of the $\gamma_{\mathrm{SINR}}, F_{\gamma_{\mathrm{SINR}}}(x)$, is given by

$$
F_{\gamma_{\text {SINR }}}(x)=\operatorname{Pr}\left(\gamma_{\text {SINR }}<x\right)=\int_{0}^{\infty} F_{S}(x(z+1)) f_{J}(z) d z .
$$

A closed-form expression for (13) is provided in the following theorem.

Theorem 1: The CDF of the receive SINR at the CDD-RX in i.n.i.d. frequency selective fading channels is given by

$$
\begin{aligned}
F_{\gamma_{\mathrm{SINR}}}(x)= & \sum_{m=1}^{M} \sum_{j=1}^{N_{h, m}}(-1)^{m} \theta_{m, j}\left(\tilde{\alpha}_{h, m}\right)^{j}\left[1-(1-\mathcal{F}) C_{1} \sum_{l=0}^{j-1} \frac{\left(\tilde{\alpha}_{h, m}\right)^{-l}}{\Gamma(l+1)} x^{l} e^{-\frac{\sigma_{z}^{2} x}{\alpha_{h, m}}} \sum_{j j=0}^{l}\left(\begin{array}{c}
l \\
j j
\end{array}\right) \Gamma\left(j j+N_{j, L}\right)\right. \\
& \left(\frac{x}{\tilde{\alpha}_{h, m}}+\frac{1}{\tilde{\alpha}_{j, L}}\right)^{-j j-N_{j, L}}-\mathcal{F} C_{2} \sum_{l=0}^{j-1} \frac{\left(\tilde{\alpha}_{h, m}\right)^{-l}}{\Gamma(l+1)} x^{l} e^{-\frac{\sigma_{z}^{2} x}{\tilde{\alpha}_{h, m}}} \sum_{j j=0}^{l}\left(\begin{array}{c}
l \\
j j
\end{array}\right) \Gamma\left(j j+N_{j, n L}\right) \\
& \left.\left(\frac{x}{\tilde{\alpha}_{h, m}}+\frac{1}{\tilde{\alpha}_{j, n L}}\right)^{-j j-N_{j, n L}}\right]
\end{aligned}
$$

where $C_{1} \triangleq \frac{1}{\Gamma\left(N_{j, L}\right)\left(\tilde{\alpha}_{j, L}\right)^{N_{j, L}}}$, and $C_{2} \triangleq \frac{1}{\Gamma\left(N_{j, n L}\right)\left(\tilde{\alpha}_{j, n L}\right)^{N_{j, n L}}}$.

Proof: Since we can readily derive (14), we omit the detailed derivation.

Note that Theorem 1 provides a general analytical framework considering a time share factor $\mathcal{F}$ for $\operatorname{LoS}$ and nLoS paths due to a possible random location of the interferer within the communication cell. Thus, this new expression for the $\mathrm{CDF}$ of the SINR can be used for a wide range of scenarios with i.n.i.d. frequency selective fading channels, arbitrary-degree of RRH cooperation via $\mathrm{dCCD}$, interference power, and communication cell size.

\section{Outage probability and diversity gain analysis}

1) Interference-Limited Region: In the interference limited region, the influence of interference is assumed to be larger than that of the noise, so that (7) can be approximated as follows:

$$
\gamma_{\text {SINR }}^{\mathrm{IL}} \approx \frac{\sum_{m=1}^{M} \tilde{\alpha}_{h, m}\left\|\tilde{\boldsymbol{h}}_{m}\right\|^{2}}{P_{J}\|\boldsymbol{j}\|^{2}}
$$

which shows that diversity gain in terms of $\frac{P_{s}}{\sigma_{z}^{2}}$ cannot be achievable when $P_{J}$ is fixed independent of the noise power.

Proposition 2: The asymptotic outage probability floor at the outage level $o_{\text {th }}$ limits the asymptotic outage probability in the interference-limited region, which is given by

$$
\begin{aligned}
P_{\text {outage }}^{\mathrm{IL}}\left(o_{\mathrm{th}}\right)= & \sum_{m=1}^{M} \sum_{j=1}^{N_{h, m}}(-1)^{m} \theta_{m, j}\left(\tilde{\alpha}_{h, m}\right)^{j}\left[1-(1-\mathcal{F}) C_{1} \sum_{l=0}^{j-1} \frac{\left(\tilde{\alpha}_{h, m}\right)^{-l}}{\Gamma(l+1)}\left(o_{\mathrm{th}}\right)^{l} \Gamma\left(l+N_{j, L}\right)\right. \\
& \left.\left(\frac{o_{\mathrm{th}}}{\tilde{\alpha}_{h, m}}+\frac{1}{\tilde{\alpha}_{j, L}}\right)^{-l-N_{j, L}}-\mathcal{F} C_{2} \sum_{l=0}^{j-1} \frac{\left(\tilde{\alpha}_{h, m}\right)^{-l}}{\Gamma(l+1)}\left(o_{\mathrm{th}}\right)^{l} \Gamma\left(l+N_{j, n L}\right)\left(\frac{o_{\mathrm{th}}}{\tilde{\alpha}_{h, m}}+\frac{1}{\tilde{\alpha}_{j, n L}}\right)^{-l-N_{j, n L}}\right] .
\end{aligned}
$$


Proof: This can be readily obtained from Theorem 1 .

We can see that $P_{\text {outage }}^{\mathrm{IL}}\left(o_{\mathrm{th}}\right)$ does not have noise power, so that this proposition verifies that the dCDD-based CPSC system cannot achieve a diversity gain in the interference-limited region, whereas it is achievable only in the noise-limited region [1]. Parameters, such as the degrees of RRH cooperation, number of multipath components, interference power, time-share factor for LoS and nLoS paths, size of the communication cell, and pathloss exponent jointly determine the floor on the outage probability.

2) Noise-Limited Region: In this region, the SINR is approximated as follows:

$$
\gamma_{\mathrm{SLINR}}^{\mathrm{NL}} \approx \frac{\sum_{m=1}^{M} \tilde{\alpha}_{h, m}\left\|\tilde{\boldsymbol{h}}_{m}\right\|^{2}}{\sigma_{z}^{2}}
$$

so that $P_{\text {outage }}^{\mathrm{NL}}\left(o_{\mathrm{th}}\right)$ can be evaluated as follows:

$$
P_{\text {outage }}^{\mathrm{NL}}\left(o_{\text {th }}\right)=\sum_{m=1}^{M} \sum_{j=1}^{N_{h, m}}(-1)^{m} \breve{\theta}_{m, j}\left(\breve{\alpha}_{h, m}\right)^{j}\left[1-\sum_{l=0}^{j-1} \frac{\left(\breve{\alpha}_{h, m}\right)^{-l}}{\Gamma(l+1)}\left(o_{\text {th }}\right)^{l} e^{-\frac{o_{\text {th }}}{\alpha_{h, m}}}\right]
$$

where $\breve{\alpha}_{h, m} \triangleq \frac{\tilde{\alpha}_{h, m}}{\sigma_{z}^{2}}$, and $\breve{\theta}_{m, j}$ can be obtained from $\theta_{m, j}$ by replacing $\tilde{\alpha}_{h, m}$ with $\breve{\alpha}_{h, m}$.

\section{Simulations}

The following simulation setting is considered:

- $B=320$ and $N_{p}=64$, so that $K=5$ is the maximum number of RRHs for dCDD.

- Quadrature phase-shift keying (QPSK) modulation is used.

- Path-loss exponents are respectively assumed to be $\epsilon_{\mathrm{L}}=2.09$, and $\epsilon_{\mathrm{nL}}=3.75$, for LoS and nLoS paths [15].

- The transmission power is assumed to be $P_{s}=1$ at all RRHs.

- As a particular example, we assume a fixed number of multipath components over the interfering channels with $N_{j, \mathrm{~L}}=2$ and $N_{j, \mathrm{~nL}}=3$.

- The CDD-RX and the interferer are respectively placed at $\left(x_{R}, y_{R}\right)$ and $\left(x_{I}, y_{I}\right)$ with respect to the center of a circular shaped communication cell of radius $R$. In the simulation, we assume that $\left(x_{R}, y_{R}\right)=[1,3]$ and $\left(x_{I}, y_{I}\right)=[0.5,5]$.

The curves obtained via link-level simulations are denoted by Ex. Analytical performance curves are denoted by An. The SNR threshold causing an outage is fixed at $o_{\mathrm{th}}=1 \mathrm{~dB}$.

\section{A. Outage Probability}

In Fig. 2, we verify the derivation for the outage probability for various cases. We assume $M=2$ and $M=4$ RRHs in the system, wherein all the RRHs employ dCDD under the control of the CU. For this particular simulation scenario, we assume that $R=10$ and $J=0 \mathrm{~dB}$. We can observe from this figure that as $M$ increases a lower outage probability can be obtained due to an increase in the received signal power at the CDD-RX. However, as $P_{s} / \sigma_{z}^{2}$ increases, the outage probability approaches the outage limits respectively specified by $P_{\text {outage }}^{\mathrm{IL}}\left(o_{\mathrm{th}}\right), M=2$ and $P_{\text {outage }}^{\mathrm{IL}}\left(o_{\mathrm{th}}\right), M=4$. For different degrees of RRH cooperation, we can see a good agreement between the exact outage probability and that of the analytic derivation. Thus, in the sequel, we will mainly use the analytical expression to evaluate the performance of the proposed system for various scenarios. 


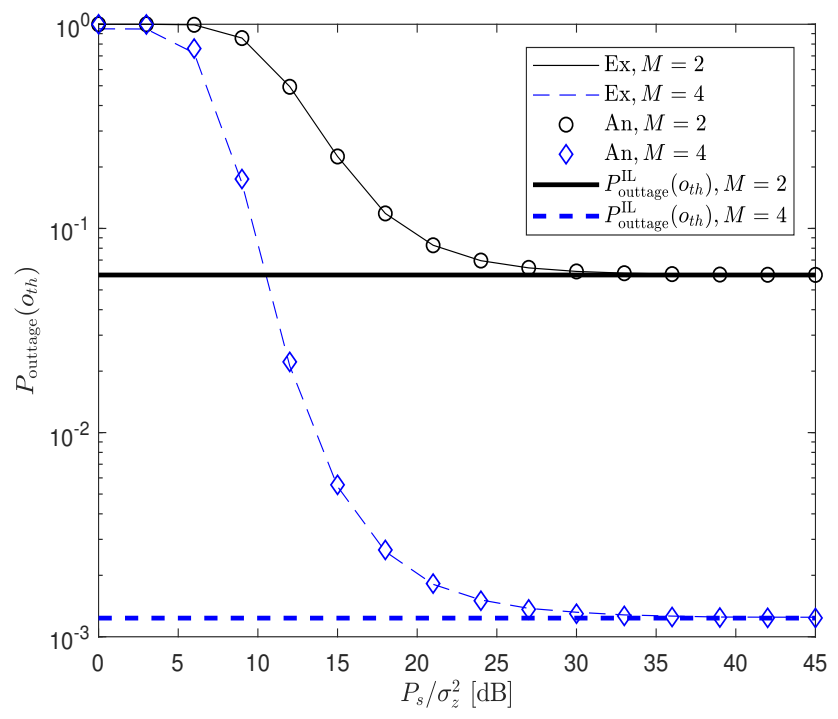

Fig. 2. Outage probability for various scenarios at a fixed value of $N_{h} s=\{1,2,3,2\}$ and $\left(N_{j, \mathrm{~L}}=2, N_{j, \mathrm{~nL}}=3\right)$.

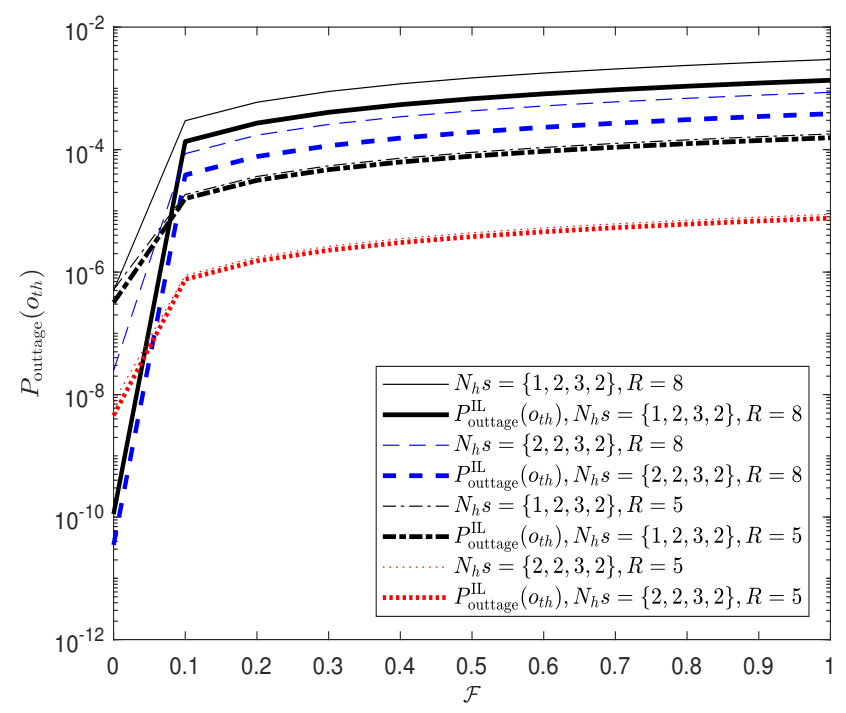

Fig. 3. Outage probability for various scenarios at a fixed value of $M=4$ and $J=0 \mathrm{~dB}$.

In Fig. 3, at a fixed value of $\left(M=4, P_{J}=0 \mathrm{~dB}, \frac{P_{s}}{\sigma_{z}^{2}}=15 \mathrm{~dB}\right)$, we investigate the impact of several system parameters on the outage probability, for example, the number of multipath components over the channels from the RRHs to the CDD-RX, different size of the communication cell, $R$, and time sharing factor $\mathcal{F}$. As $\mathcal{F}$ increases, the system experiences the $\mathrm{nLoS}$ path more often, so that a higher outage probability is obtained due to a greater number of multipath components. In general, as the number of multipath components over the channels from the 
RRHs to the CDD-RX increases, a lower outage probability is obtained due to an increased receive signal power at the CDD-RX. Also, we can see that the cell size has a negative impact on the outage probability since the receive signal power is affected by the distance from the RRHs to the CDD-RX. As either communication cell is increased or the number of multipath components over the channels from the RRHs to the CDD-RX is increased, a lower outage probability is obtained.

To investigate the diversity gain in detail, we use various values of $\mathcal{F}$ and $N_{h}$ s for the system with $M=2$. From

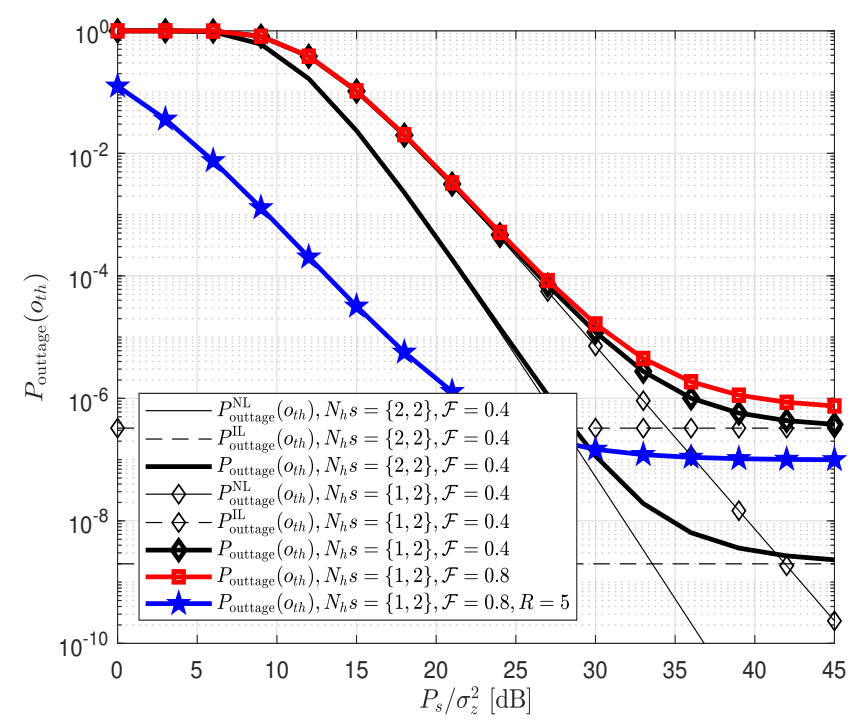

Fig. 4. Diversity gain analysis for various scenarios with $J=-15 \mathrm{~dB}$ and $M=2$. When there is no specification about $R$, then we assume $R=10$.

Fig. 4, we can summarize the following observations.

- The interference-limited region is noticeably different from the noise-limited region. As $\frac{P_{s}}{\sigma_{z}^{2}}$ increases, the outage probability approaches $P_{\text {outage }}^{\mathrm{IL}}\left(o_{\text {th }}\right)$.

- In the interference-limited region, the outage probability is dominated by $P_{\text {outage }}^{\mathrm{IL}}\left(o_{\mathrm{th}}\right)$, and the slope of the outage probability becomes zero. That is, the diversity gain is not achievable.

- The maximum diversity gain, $G_{d}=N_{h, 1}+N_{h, 2}$, is achievable only in the noise-limited region [1], [21], which is dependent on the number of RRHs being selected for dCDD, and the number of multipath components over a particular frequency fading channel. Thus, the diversity gain depends on the total number of dCDD RRHs in the system. Although the time sharing parameter, $\mathcal{F}$, influences the limit on the outage probability, it has no impact on the diversity gain in the noise-limited region. In addition, the communication cell, $R$, has significant impact on the outage probability since the receive power is dictated by the distance from the RRH to the CDD-RX. However, it has no impact on the diversity gain in the noise-limited region. 


\section{CONCLusions}

In this paper, we have investigated the effects of the interferer and i.n.i.d frequency selective fading with coexisting LoS and nLoS paths on the outage probability of a single carrier system that applies dCDD. For a more realistic channel setting, we have derived a new form of the CDF of the SINR, and then derived closed-form expressions for the outage probability. From the derivation and system level simulations, we have verified the existence of an interference-limited region, in which a diversity gain is not achievable. In contrast, in the noise-limited region, the achievable diversity gain has been shown to be the sum of the multipath components across the channels from the RRHs to the CDD-RX.

\section{REFERENCES}

[1] K. J. Kim, M. D. Renzo, H. Liu, P. V. Orlik, and H. V. Poor, "Performance analysis of distributed single carrier systems with distributed cyclic delay diversity," IEEE Trans. Commun., vol. 65, no. 12, pp. 5514 - 5528, Dec. 2017.

[2] K. J. Kim, H. Liu, M. D. Renzo, P. V. Orlik, and H. V. Poor, "Secrecy analysis of distributed CDD-based cooperative systems with deliberate interference," IEEE Trans. Wireless Commun., vol. 17, no. 12, pp. 7865-7878, Dec. 2018.

[3] IEEE, "Wireless LAN medium access control (MAC) and physical layer (PHY) specifications-Amendment 4: Enhancements for very high throughput for operation in bands below $6 \mathrm{GHz}, "$ IEEE Standard 802.11ac-2013, Part 11, 2009.

[4] — - "Wireless LAN medium access control (MAC) and physical layer (PHY) specifications - Amendment 5: Enhancements for higher throughput," IEEE Standard 802.11n-2009, Part 11, 2009.

[5] 3GPP, Technical Specification Group Radio Access Network, "Evolved universal terrestrial radio access (E-UTRA): Physical channels and modulation (release 8)," 3GPP TS 36.211 V8.9.0 (2009-12) Technical Specification, Mar. 2009.

[6] Y.-C. Liang, W. S. Leon, Y. Zeng, and C. Xu, "Design of cyclic delay deievsity for single carrier cyclic prefix (sccp) transmissions with block-iterative GDFE (BI-GDFE) receiver," IEEE Trans. Wireless Commun., vol. 7, no. 2, pp. 677-684, Feb. 2008.

[7] A. H. Mehana and A. Nosratinia, "Single-carrier frequency-domain equalizer with multi-antenna transmit diversity," IEEE Trans. Wireless Commun., vol. 12, pp. 388-397, Jan. 2013.

[8] Q. Li, Q. Yan, K. C. Keh, K. H. Li, and Y. Hu, "A multi-relay-selection scheme with cyclic delay diversity," IEEE Commun. Lett., vol. 17, no. 2, pp. 349-352, Feb. 2013.

[9] U.-K. Kwon and G.-H. Im, "Cyclic delay diversity with frequency domain turbo equalization for uplink fast fading channels," IEEE Commun. Lett., vol. 13, no. 3, pp. 184-186, Mar. 2009.

[10] K. J. Kim and T. A. Tsiftsis, "On the performance of cyclic prefix-based single-carrier cooperative diversity systems with best relay selection," IEEE Trans. Wireless Commun., vol. 10, no. 4, pp. 1269-1279, Apr. 2011.

[11] K. J. Kim, T. A. Tsiftsis, and H. V. Poor, "Power allocation in cyclic prefixed single-carrier relaying systems," IEEE Trans. Wireless Commun., vol. 10, no. 7, pp. 2297-2305, Jul. 2011.

[12] X. Zhang and J. G. Andrew, "Downlink cellular network analysis with multi-slope path loss models," IEEE Trans. Commun., vol. 63, no. 5, pp. 1881-1894, May 2015.

[13] T. Bai, R. Vaze, and R. W. Heath, "Analysis of blockage effects on urban cellular networks," IEEE Trans. Wireless Commun., vol. 9, no. 13, pp. 5070-5083, Sep. 2014.

[14] M. Ding, P. Wang, D. Lopez-Perez, G. Mao, and Z. Lin, "Performance impact of LoS and NLoS transmissions in dense cellular networks," IEEE Trans. Wireless Commun., vol. 15, no. 3, pp. 2365-2380, Mar. 2016.

[15] 3GPP, TR 36.828 (V11.0.0), "Further enhancements to lte time division duplex (TDD) for downlink-uplink (DL-UL) interference management and traffic adaptation,” Jun. 2012.

[16] C. M. Lo and W. H. Lam, "Performance of generalized selection combining for mobile radio communications with mixed cochannel interferers," IEEE Trans. Veh. Technol., vol. 51, no. 1, pp. 114-121, Jan. 2002.

[17] Y. Zeng and T. S. Ng, "Pilot cyclic prefixed single carrier communication: channel estimation and equalization," IEEE Signal Process. Lett., vol. 12, no. 1, pp. 56-59, Jan. 2005.

[18] F. Gao, A. Nallanathan, and C. Tellambura, "Blind channel estimation for cyclic-prefixed single-carrier systems by exploiting real symbol characteristics,” IEEE Trans. Veh. Technol., vol. 56, no. 5, pp. 2487-2498, Sept. 2007. 
[19] K. J. Kim, Y. Yue, R. A. Iltis, and J. D. Gibson, "A QRD-M/Kalman Filter-based detection and channel estimation algorithm for MIMOOFDM systems," IEEE Trans. Wireless Commun., vol. 4, pp. 710-721, Mar. 2005.

[20] X. W. Cui, Q. T. Zhang, and Z. M. Feng, "Outage performance for maximal ratio combiner in the presence of unequal-power co-channel interferers,” IEEE Commun. Lett., vol. 8, no. 5, pp. 289-291, May 2004.

[21] K. J. Kim, M. D. Renzo, H. Liu, P. V. Orlik, and H. V. Poor, "Diversity gain analysis of distributed CDD systems in non-identical frequency selective fading," in Proc. IEEE Int. Conf. Commun., Kansas City, MO, USA, May 2018. 